\title{
PERMUTATIONS PRESERVING CONVERGENCE OF SERIES
}

\section{RALPH PALMER AGNEW}

1. Introduction. We shall solve a problem involving rearrangements of series which was proposed to the author by R. Creighton Buck. The problem is to characterize those permutations $p_{1}, p_{2}, p_{3}, \cdots$ of the integers $1,2,3, \cdots$ such that the two series in

$$
\sum_{k=1}^{\infty} a_{p_{k}}=\sum_{k=1}^{\infty} a_{k}
$$

converge to equal values whenever $\sum a_{k}$ is a convergent series of real (or complex) terms. We shall show that a given permutation $p_{1}, p_{2}, \cdots$ has the required property if and only if there is an integer $N$ such that for each $n=1,2,3, \cdots$ the set of integers which appear among the integers $p_{1}, p_{2}, \cdots, p_{n}$ is representable as the union of $N$ or fewer blocks of consecutive integers.

2. Proof. Let $p_{1}, p_{2}, \cdots$ be a given permutation of the integers $1,2,3, \cdots$. Let

$$
s(n)=\sum_{k=1}^{n} a_{k}, \quad \sigma(n)=\sum_{k=1}^{n} a_{p_{k}} .
$$

Suppose that $n$ is so great that the integer 1 is included in the set $p_{1}, p_{2}, \cdots, p_{n}$. Then the integers in the set $p_{1}, p_{2}, \cdots, p_{n}$ are, in increasing order,

$$
\begin{gathered}
1,2,3, \cdots, \beta_{0}^{(n)}, \alpha_{1}^{(n)}+1, \alpha_{1}^{(n)}+2, \cdots, \beta_{1}^{(n)}, \alpha_{2}^{(n)}+1, \\
\alpha_{2}^{(n)}+2, \cdots, \beta_{2}^{(n)}, \cdots, \alpha_{j_{n}}^{(n)}+1, \alpha_{j_{n}}^{(n)}+2, \cdots, \beta_{j_{n}}^{(n)}
\end{gathered}
$$

where

$$
0<\beta_{0}^{(n)}<\alpha_{1}^{(n)}<\beta_{1}^{(n)}<\alpha_{2}^{(n)}<\beta_{2}^{(n)}<\cdots<\beta_{j_{n}}^{(n)} .
$$

Hence

$$
\sigma(n)=s\left(\beta_{0}^{(n)}\right)+\left[s\left(\beta_{1}^{(n)}\right)-s\left(\alpha_{1}^{(n)}\right)\right]+\cdots+\left[s\left(\beta_{j_{n}}^{(n)}\right)-s\left(\alpha_{j_{n}}^{(n)}\right)\right]
$$

and therefore

$$
\sigma(n)=s\left(\beta_{0}^{(n)}\right)-s\left(\alpha_{1}^{(n)}\right)+s\left(\beta_{1}^{(n)}\right)-\cdots-s\left(\alpha_{j_{n}}^{(n)}\right)+s\left(\beta_{j_{n}}^{(n)}\right) .
$$

Since $\lim _{n \rightarrow \infty} \beta_{0}^{(n)}=\infty$, this has the standard form

Received by the editors August 20, 1954. 


$$
\sigma(n)=\sum_{i=1}^{\infty} a_{n k} s(k)
$$

where ${ }^{\circ}$

$$
\lim _{n \rightarrow \infty} a_{n k}=0, \quad k=1,2,3, \cdots,
$$

and

$$
\sum_{k=1}^{\infty} a_{n k}=1, \quad n=1,2,3, \cdots .
$$

This transformation from $s(n)$ to $\sigma(n)$ is regular, that is, such that existence of $\lim s(n)$ implies $\lim \sigma(n)=\lim s_{n}$, if and only if there is a constant $M$ for which

$$
\sum_{k=1}^{\infty}\left|a_{n k}\right| \leqq M
$$

We see that

$$
\sum_{k=1}^{\infty}\left|a_{n k}\right|=2 B_{n}-1
$$

where $B_{n}$ is the number of disjoint blocks of consecutive integers appearing in the set $p_{1}, p_{2}, \cdots, p_{n}$ and hence that existence of $\lim s(n)$ implies $\lim \sigma(n)=\lim s(n)$ if and only if there is a constant $M$ for which $2 B_{n}-1 \leqq M$ or $B_{n} \leqq(M+1) / 2$. This proves the result.

Cornell University 